\title{
A Design of Passband-chosen Complex Filter and Automatic Calibration System
}

\author{
Xi Zeng ${ }^{1, a}$, Weidong Geng ${ }^{1, b^{*}}$ Yunqian Zhang $^{1, c}$ and Wenshen Wang ${ }^{2, d}$ \\ ${ }^{1}$ Institute of Photo-electronics Thin Film Devices and Technique of Nankai University , \\ Key Laboratory of Photo-Electronics Thin Film Devices and Technique of Tianjin , \\ Key Laboratory of Opto-electronic Information Science and Technology, Nankai University,Tianjin \\ 300071, China; \\ ${ }^{2}$ Beijing Langboxinwei Electricon Company, Beijing 100084, China. \\ azengxi1213@126.com, b'gengwd@nankai.edu.cn, czhangyq_nku@163.com,
}

*Corresponding author: Weidong Geng: gengwd@nankai.edu.cn

\section{Keywords: Complex Filter, Low Power Design, Calibration, Passband-chosen}

\begin{abstract}
A low power design of active RC complex filter was proposed by using 130nm CMOS process. The filter has 4 center frequencies, $100 \mathrm{kHz}, 200 \mathrm{kHz}, 300 \mathrm{kHz}$ and $500 \mathrm{kHz}$. The image rejection is higher than $40 \mathrm{~dB}$. An automatic calibration system with dichotomy algorithm and RC oscillator circuit was used to caliber both the center frequency and the band-wide. The circuit consumption of the filter and the calibration system is less than $500 \mathrm{uA}$ with $1.5 \mathrm{Vsupply}$ voltage and the calibration error is less than $1 \%$.
\end{abstract}

\section{Introduction}

Low-IF wireless communication receiver has attracted great attention of recent research and development with the advantage of avoiding DC offset. However, the signals from the mixer of the low-IF receiver has been mixed with mirror signals ${ }^{[1]}$, which are different to be distinguished with the normal signals, resulting in a great interference. The popular solution to reject the mirror signals is to use a complex filter ${ }^{[2]}$. Besides, most wireless communication devices work with internal battery-powered, so low power design of complex filters becomes a hot research spot. In reference [3], a 3-orders active RC complex filter with an integrator and a digital circuit has a bandwidth of $20 \mathrm{MHz}$, a center frequency of $46 \mathrm{MHz}$, and its current consumption is less than $7.9 \mathrm{~mA}$ with the power supply of $1.8 \mathrm{~V}$. In reference [4], a 12-orders complex filter with controllable transconductance has bandwidths of $0.5 \sim 1.5 \mathrm{MHz}$ and $1 \sim 3 \mathrm{MHz}$, center frequency of $1 \mathrm{MHz}$ and $2 \mathrm{MHz}$ and its power consumption is $2.77 \mathrm{~mW}$. Reference [5] shows a 3-orders RC complex filter applying for ZigBee, whose bandwidth is $3.09 \mathrm{MHz}$, center frequency is $2 \mathrm{MHz}$ and current supply is $1.2 \mathrm{~mA}$ with the working voltage of $1.8 \mathrm{~V}$. Traditional complex filters provide only one passband or two passbands, resulting in the application of the chips limited. Based on the SMIC130nm, this paper has proposed a 4-passbands complex filter applying for RFID, shown a capacitor array with miller compensation in order to keep the stability of the circuit and given a RC calibration module to solve the craft error.

\section{Design of Complex Filter}

\subsection{Circuit of Complex Filter}

The active RC filter has a lower noise, large output range and better stability ${ }^{[6][7]}$, which was used in this paper. For multi-stage complex filter, how integrators make multi-stage filter influences its dynamic range and the accuracy, among which filter made of the leapfrog structure has a smaller parasitic sensitivity and bigger dynamic range ${ }^{[8]}$, chosen by the Butterworth filter in this paper. Besides, the more the order of the filter is, the higher its image rejection is and the more the power consumption is, in certain center frequency and bandwidth. In order to achieve the request of the 
image rejection of $40 \mathrm{~dB}$, the complex filter in this paper uses 3-order leapfrog Butterworth structure for I/Q signal channel. The circuit of the complex filter shown as Fig.1, which is made of two identical 3-order leapfrog Butterworth LPF with 4 bandwidth, $60 \mathrm{kHz}, 120 \mathrm{kHz}, 200 \mathrm{kHz}$ and 275 $\mathrm{kHz}$. Under the role of the frequency resistors $R_{i f}$, connected between I channel and Q channel, the center frequency is changed from 0 to the target center frequency ${ }^{[9]}$.

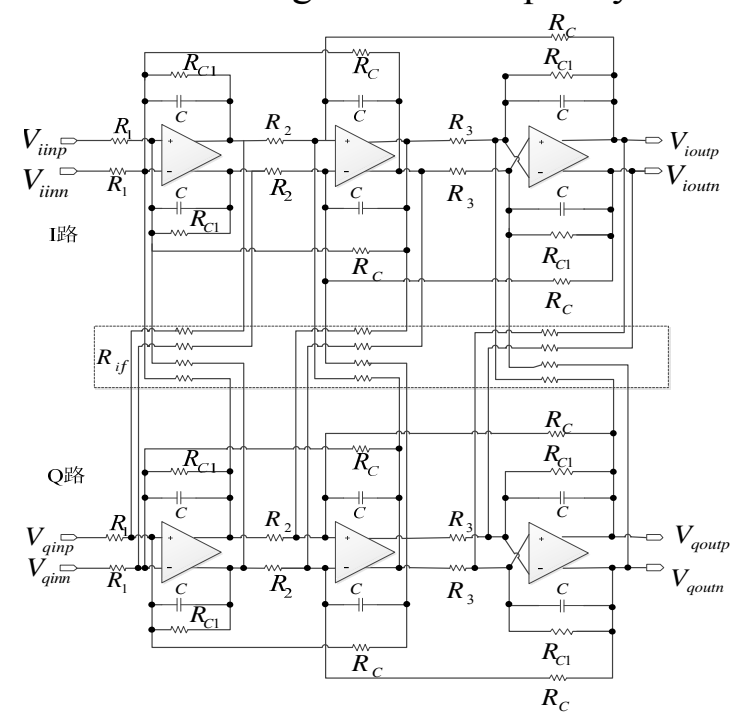

\subsection{Design of Amplifier}

Fig.1 3-order leapfrog complex filter

Amplifier is the key unit of the active RC filter, whose performance, especially the GBW ${ }^{[10]}$, determines the capability of the filter directly. The GBW must be 50 100 times of its cutoff frequency from experience. The most bandwidth of the filter proposed in this paper is $275 \mathrm{kHz}$, so the least GBW of the amplifier used in the circuit is $13.75 \mathrm{MHz}$. In order to obtain high gain and high load drive capability in the case of small layout area and power consumption, two-stage amplification structure is used in this paper. From the simulation result shown as Fig.2, it is seen that the GBW of the amplifier is $32 \mathrm{MHz}, \mathrm{PM}$ is $69.24^{\circ}$ and $\mathrm{DC}$ gain reaches $72 \mathrm{~dB}$, satisfy the requirement of the filter.

The ac simulation of the final circuit at tt corner is given as Fig.3, from which it is seen that the design of the circuit fulfill the design request.

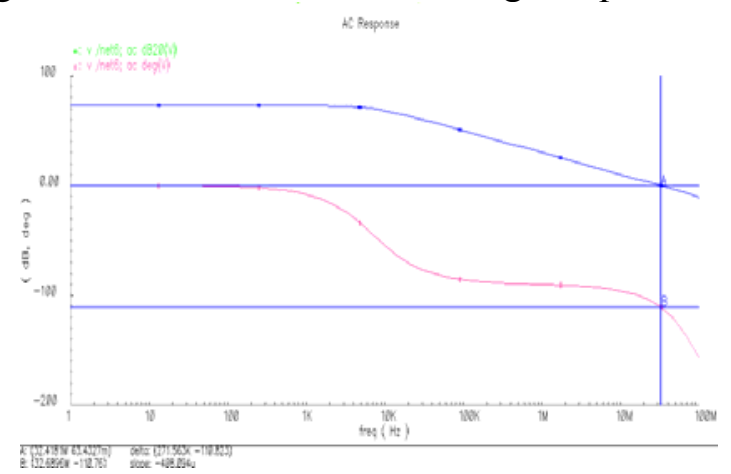

Fig.2 ac simulation of the amplifier

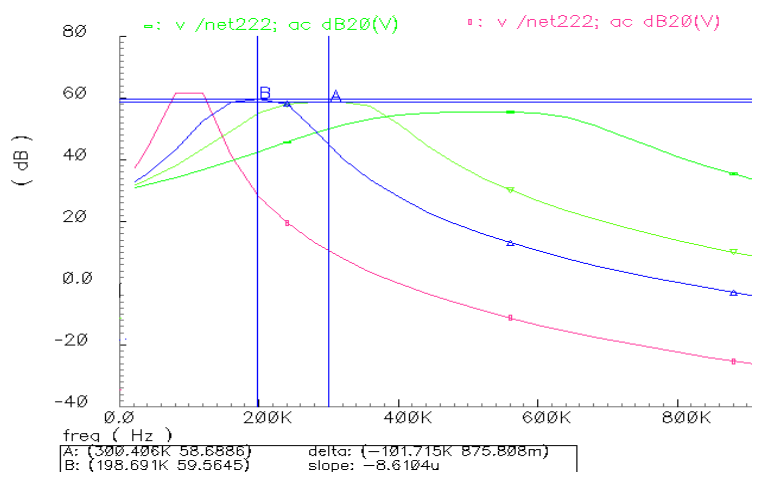

Fig. 3 ac simulation of the complex filter

\section{Design of automatic calibration system}

The bandwidth and the center frequency of the active RC complex filter are decided by resistors and capacitors, both values of which are easily influenced by temperature and process ${ }^{[11]}$, bringing in the distortion of the center frequency and bandwidth. In order to reduce the distortion, the resistor arrays used in the filter are controlled by automatic calibration system to make sure that however the process and temperature changes, the multiplier of RC, as well as the center frequency and bandwidth, stay the same value. There are two methods of calibration, front calibration and background calibration, according to the order of the calibration. On the consideration of low power design, this paper takes the measure of front calibration system shown as Fig.4, which is made up of RC oscillator 
circuit and digital module.

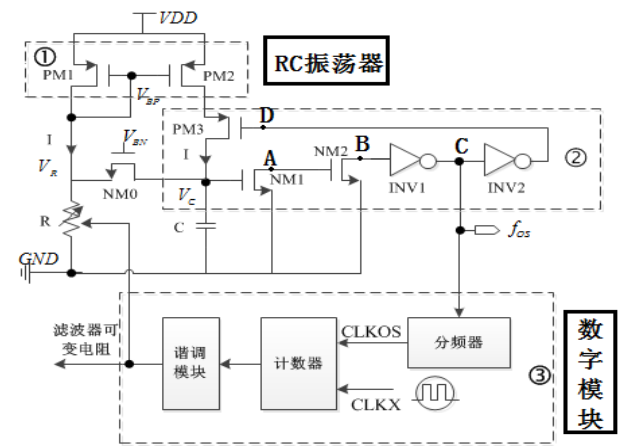

\subsection{RC Oscillator Circuit}

Fig.4 Schematic diagram of RC calibration system

The RC oscillator circuit shown in Fig.4 is made up of a self-oscillator circuit, a current mirror circuit and left comparison section. The self-oscillator circuit provides the needed current I and the bias voltages, $V_{B N}$ and $V_{B P}$ of the total circuit. And the mirror circuit, copying the current of the resistor $\mathrm{R}$ to the capacitor branch to provide current for the capacitor to charge or discharge, could be simplified to the part shown as (1) in Fig.4. In the comparison section given as (2) in Fig.4, when the voltage $V_{C}$ across the capacitor is higher than the threshold voltage, namely $V_{C} \mathbf{z} V_{G S 1}-V_{W_{1} 1}$, MOS NM1 turns on, then the voltage of point A turns down and the MOS NM2 turns off, resulting in the voltage of point B to turn high. After inverters INV1 and INV2, the voltage of point D turns high and the MOS PM3 turns off, thus the capacitor will discharge to variable resistor $\mathrm{R}$ through the MOS NM0. On the contrary, when $V_{c}^{E}<V_{G S 1}^{T}-V_{T H a}$, NM1 turns off and the capacitor will be charged by current through PM3 from PM2. When the capacitor is charged and discharged continuously by the oscillator, the output signal will be square wave signal with the frequency of $f_{\text {OS }}$.

During the charging and discharging time of $\Delta t$ the changing voltage of the capacitor is $\Delta V_{G}$. Supposing that the current flowing through PM1 is $I$, the current flowing through PM2 is $I$ as well under the influence of the current mirror. The variable parameters meet the function below.

$$
\Delta V_{C}=\frac{I \bullet \Delta t}{C}
$$

The voltage across the variable resistor $\mathrm{R}$ is $V_{R}$, then

$$
I=\frac{V_{R}}{R}
$$

From function (2) and function (3), the function (4) is easily known.

$$
\Delta t=R C \bullet \frac{\Delta V_{C}}{V_{R}}
$$

The charging and discharging cycle time of the capacitor is obtained as function (5).

$$
T=2 \Delta t=2 R C \bullet \frac{\Delta V_{C}}{V_{R}}
$$

The frequency of the output signal of the RC oscillator is given as function (6).

$$
f_{o s}=\frac{1}{T}=\frac{V_{R}}{2 R C \Delta V_{C}}
$$

\subsection{Digital Automatic Calibration Module}

The digital automatic calibration module is parted into two sub-modules, the frequency counting module and the comparison module. The output signal of the RC oscillator is divided by two. Taking the stable square wave CLKX generated by crystal as a standard, the signal CLKOS divided from the output signal of the oscillator is counted and ratio $N$ between CLKOS and CLKX fulfill the 
function $f_{o s}=\frac{N \bullet f_{X}}{2}$. In the comparison module, the control to the resistor array is implemented by comparing $f_{o s}$ and the reference signal $f_{R E F}$.

The frequency of the reference signal is $32 \mathrm{kHz}$, the simulation at the corner of $\mathrm{tt} / \mathrm{ff} / \mathrm{ss}$ is given as Fig.6. From the figure, it is seen that the output frequencies of the 3 different corners are $32.10 \mathrm{kHz}$, $32.38 \mathrm{kHz}$ and $31.26 \mathrm{kHz}$. The error is less than $2.5 \%$.

\section{Simulation result and layout}

The circuit is simulated on SMIC 130nm. It is demonstrated that the calibration succeeds and the control code of the resistor array will be saved in the register after finishing the automatic calibration process. Then when the complex filter starts to work, the code will be sent into successfully and the calibration of the filter will be complemented. As an example of $300 \mathrm{kHz}$ mode, the simulation curve is given as 1 in Fig. 5 with the center frequency of $300 \mathrm{kHz}$ and the bandwidth of $278 \mathrm{kHz}$, satisfying the design request. If the circuit isn't calibrated, the curves of the ac simulation on ss and ff corners will be 2 and 5 in Fig. 5 with the error of the center frequency more than 50\%. On the contrast, the error will be less than 1\% after the calibration with the curves shown as 3 and 4 in Fig.5. The circuit works on the supply voltage of $1.5 \mathrm{~V}$ and the current of less than $500 \mathrm{uA}$, that is to say, the total power consumption is less than $0.75 \mathrm{~mA}$.

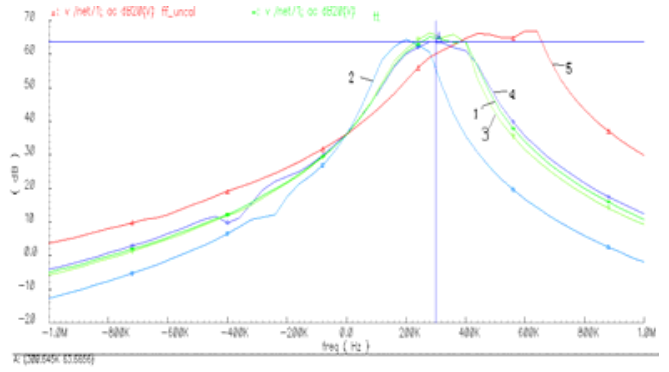

Fig. 5 ac simulation of the filter on $300 \mathrm{kHz}$ mode

The contrast of the power consumption, the orders and the image rejection of the complex filters shown in different paper are given in list 1 , from which it is easily seen that the complex filter proposed in this paper has the advantage of multi-passband and low power consumption.

Table1 Contrast of the results

\begin{tabular}{ccccc}
\hline & This paper & reference[3] & reference [4] & reference [5] \\
\hline Power(mW) & 0.75 & 1.16 & 2.77 & 14.22 \\
\hline Order & 3 & 3 & 12 & 7 \\
\hline Image rejection $(\mathrm{dB})$ & 45 & 47.5 & 71 & 98 \\
\hline Passband amount & 4 & 1 & 2 & 1
\end{tabular}

Fig.6 shows the total layout of the chip with the total area of $3.4 \mathrm{~mm} * 3.4 \mathrm{~mm}$. In the center of the layout is the complex filter and the upper part, shown as rectangle 1, is the analog circuit, namely RC oscillator circuit. The left and the bottle part is the digital module of the chip, which contains the digital automatic calibration system of the filter.

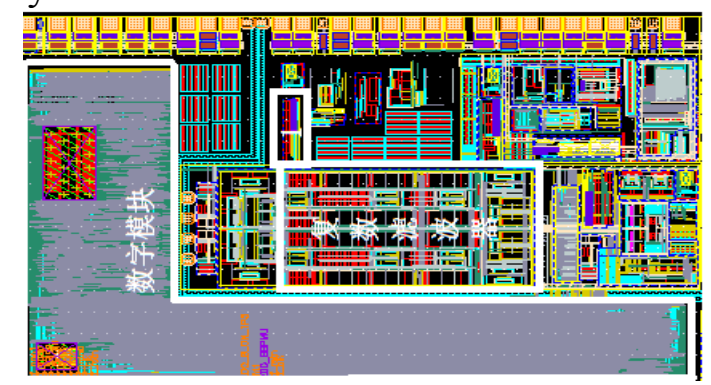

Fig.6 Layout of the total chip

\section{Summary}

A low power design of complex filter with 4 pass bands has been proposed in this paper. All of the capacitor arrays used in the circuit have the structure of miller compensation to solve the problem of 
instability resulting from multi-band and multi-poles. In order to compensate the difference of the center frequency and bandwidth generated by the process, an automatic calibration system made up of a RC oscillator and digital module has been present. The complex filter shown this paper works at the current less than $500 \mathrm{uA}$ at the supply voltage of $1.5 \mathrm{~V}$ while its image rejection reaches more than $40 \mathrm{~dB}$ and the difference is less than $1 \%$, applying for RFID chips properly. The filter have 4 pass bands, which means wider application prospects.

\section{References}

[1] Du Dingkun, Li Yongming, Wang Zhihua. Design of a 4MHz Complex Filter with Automatic Tuning System[J]. Microelectronics.2006,36（6）: 820-824;

[2] Sheng W, Xia B, Emira A ect. A 3V 0.35um COMS Bluetooth receiver IC. IEEE Journal of Solid State circuits, in RFIC Symp. Jun. 2002, pp. 107-110.

[3] Zeng Jianping, Hu Jiajie, Shan Qiang al ect. A Design of 46MHz Gm-C Complex Filter and Automatic Frequency Tuning Circuit[J]. Journal of Hunan University(Natural Sciences), 2014,08, 41(08): 84-88.

[4] Richa Arya, George Souliotis, Spyros Vlassis,Costas Psychalinos. A 0.5 V Tunable Complex Filter for Bluetooth and Zigbee Using OTAs[J]. Analog Integr Circ Sig Process. 2014,79:73-81.

[5] Li Di, Jing Zhang, Yang Yintang etc. Third-order Active-RC Complex Filter with Automatic Frequency Tuning for ZigBee Transceiver Applications[J], J.Cent,South Univ, 2015:pp. 966-7973

[6] Wei Junchen, Yin Yadong. An Active Image Rejection Complex Filter Design[J]. Microelectronics and Computer, 2014,01,31 (1): 148-155.

[7] HARRISON J. WESTE N. $350 \mathrm{MHz}$ Opamp-RC Filter in 0.18um CMOS[J]. Electronics Letters, 2002,38(6): 259-260.

[8] H W Su. Y Sun, R Gordon.Performance Analysis and Comparison of High Frequency CMOS OTA-C Filter[A].IEE Seminaron Analog Signal Processing[C].Oxford,UK, ${ }^{\text {st }}$ Nov.2000:8/1-8/7.

[9] Du Dingkun, Li Yongming, Wang Zhihua ,ect. An Active-RC Complex Filter with Mixed Signal Tuning System for Low-IF Receiver[C]//IEEE APCCAS 2006 [s.1.]:IEEE,2006:1031-1034.

[10] C Cuypers, NY Voo M etc. Genaral Synthesis of Complex Analogue Filters. IEE Proc Circuits Devices Syst,2005:pp 7-15.

[11] CHEN Gang, Li Zhiqun, SU Haiyong ect. A5th-order Chebyshev Active RC Complex Filter with Automatic Frequency Tuning for Wireless Sensor Networks Application[C] //2010 International Symposium on Signals Systems and Electronics Nanjing:ISSSE,2010:1-4. 